Note

\title{
The Population Genetics of Evolutionary Rescue in Diploids: X Chromosomal versus Autosomal Rescue
}

\author{
Robert L. Unckless ${ }^{1, \star}$ and H. Allen Orr ${ }^{2}$ \\ 1. Department of Molecular Biosciences, University of Kansas, Lawrence, Kansas 66045; 2. Department of Biology, University of \\ Rochester, Rochester, New York 14627 \\ Submitted February 12, 2019; Accepted August 16, 2019; Electronically published January 15, 2020 \\ Online enhancements: appendix figure.
}

\begin{abstract}
Most population genetic theory assumes that populations adapt to an environmental change without a change in population size. However, environmental changes might be so severe that populations decline in size and, without adaptation, become extinct. This "evolutionary rescue" scenario differs from traditional models of adaptation in that rescue involves a race between adaptation and extinction. While most previous work has usually focused on models of evolutionary rescue in haploids, here we consider diploids. In many species, diploidy introduces a novel feature into adaptation: adaptive evolution might occur either on sex chromosomes or on autosomes. Previous studies of nonrescue adaptation revealed that the relative rates of adaptation on the $\mathrm{X}$ chromosome versus autosomes depend on the dominance of beneficial mutations, reflecting differences in effective population size and the efficacy of selection. Here, we extend these results to evolutionary rescue and find that, given equal-sized chromosomes, there is greater parameter space in which the $\mathrm{X}$ is more likely to contribute to adaptation than the autosomes relative to standard nonrescue models. We also discuss how subtle effects of dominance can increase the chance of evolutionary rescue in diploids when absolute heterozygote fitness is close to 1 . These effects do not arise in standard nonrescue models.
\end{abstract}

Keywords: adaptation, autosomes, environmental change, evolutionary rescue, sex chromosomes.

Sex chromosomes evolve differently from autosomes, and this has consequences for molecular evolution, the appearance and evolution of novel genes, the accumulation of reproductive isolation between populations, and several other processes (Betrán et al. 2002; Vicoso and Charlesworth 2006; Mank et al. 2007, 2010; Presgraves 2008; Hollinger and Hermisson 2017; Lasne et al. 2019). The unique nature of sex chromosome evolution is caused by unequal ploidy and therefore differing dominance relations in the two sexes. In

\footnotetext{
* Corresponding author; email: rlu1@cornell.edu, unckless@ku.edu. ORCIDs: Unckless, https://orcid.org/0000-0001-8586-7137.
}

Am. Nat. 2020. Vol. 195, pp. 561-568. (C) 2020 by The University of Chicago. 0003-0147/2020/19503-59061\$15.00. All rights reserved.

DOI: $10.1086 / 707139$ a male-heterogametic species (i.e., males are XY), X-linked mutations segregate in males one-third of the time, but they are hemizygous, and thus recessive fitness effects are fully exposed. In contrast, X-linked mutations segregate in females two-thirds of the time, but they are heterozygous, and thus recessive effects may be partly or wholly masked. These unique properties along with reduced effective mutation rates (there are $25 \%$ fewer X chromosomes than autosomes when populations have equal sex ratios) lead to different expectations about the probability and rate of adaptation on the $\mathrm{X}$ versus on the autosomes as well as differences in expectations for genetic drift, rates of gene flow between populations, and recombination (reviewed in Hedrick 2007).

Adaptation on sex chromosomes-here we specifically consider the $\mathrm{X}$ or $\mathrm{Z}$ chromosome, i.e., the chromosome that appears in both sexes - is well studied (reviewed in Vicoso and Charlesworth 2006). Two previous results are particularly relevant. First, Charlesworth et al. (1987) found that adaptation from new mutation is more likely on the $\mathrm{X}$ than autosomes if the dominance coefficient of the mutation is less than 0.5 (i.e., partially or completely recessive), assuming equally strong selection on homozygotes in both sexes. Second, Orr and Betancourt (2001) found that adaptation from the standing genetic variation is less likely on the $\mathrm{X}$ than on autosomes if alleles in the old environment were deleterious and initially segregated at mutationselection equilibrium.

These previous studies of $\mathrm{X}$ versus autosomal adaptation considered populations that are of constant size through time. More recently, the population genetics of adaptation in a population of changing size has been considered (Otto and Whitlock 1997; Uecker and Hermisson 2011). Specifically, evolutionary rescue - when a population is saved from extinction by rapid genetic adaptation to an environmental change - has received a good deal of attention (Bell 2008; Orr and Unckless 2008, 2014; Kirkpatrick and Peischl 2013; Uecker et al. 2014; Uecker and Hermisson 2015; Wilson et al. 2017). These models are typically haploid and are 
thus biased somewhat toward microbial species. This bias reflects that (i) microbes allow ready experimental tests of theoretical predictions and that (ii) models of evolutionary rescue can be applied to important phenotypes, like antibiotic and drug resistance of pathogens (Ramsayer et al. 2013). However, two recent articles explicitly modeled evolutionary rescue in diploids (Glemin and Ronfort 2013; Uecker 2017). Both consider the role of mating in rescue. Glemin and Ronfort focused on adaptation in selfing versus outcrossing species but examined rescue as a special case. Uecker focused on evolutionary rescue and expanded on the Glemin and Ronfort results, looking at clonal, selfing, and outcrossing populations.

Here, we explore evolutionary rescue when mutations that can rescue threatened populations occur on the $\mathrm{X}$ chromosome (or $\mathrm{Z}$ chromosome) versus the autosomes. We contrast these results to previous ones on adaptation in populations not threatened by extinction. We find that when evolutionary rescue occurs via new mutation, the parameter space in which the $\mathrm{X}$ is more likely to save the population than autosomes is larger in the rescue case than in the standard adaptation case. When evolutionary rescue occurs via alleles from standing genetic variation, on the other hand, autosomal rescue is usually more likely so long as the mutation is net beneficial in heterozygotes. We also note a curious nuance of evolutionary rescue in diploidswhen absolute heterozygote fitness is close to unity, evolutionary rescue may be more likely than our analytical results predict because rescue alleles can persist in a population while wild-type alleles are declining to extinction. These rescue alleles might then later form homozygotes that enjoy especially high absolute fitness. Although our analytic approach is approximate - and in some cases break down - our findings generally agree well with exact computer simulations and, at worst, provide a rough guide to the dynamics of rescue in diploids.

\section{The Model and Preliminary Considerations}

Building on previous haploid models (Bell 2008; Orr and Unckless 2008, 2014), we model a random mating diploid population with initial population size $N_{0}$. At time zero, the environment changes suddenly and the absolute fitness of the wild-type allele drops below 1 . Consequently, the number of wild-type individuals begins to decline. In our analytic work, we assume that wild-type individuals are numerous enough that this decline is deterministic with rate $r$. In our computer simulations, we relax this deterministic simplification (see below). Mutant alleles have selective advantage $h s$ in heterozygotes and $s$ in homozygotes and hemizygotes (see table 1 for a complete fitness schedule). Other than the effects of hemizygosity versus heterozygosity per se, we do not allow sex differences in fitness.
Table 1: Genotype fitness schedule after the environmental change

\begin{tabular}{llc}
\hline Genotype & Absolute fitness & Fitness excess \\
\hline $\mathrm{A}_{\mathrm{wt}} \mathrm{A}_{\mathrm{wt}}$ & $(1-r)$ & $-r$ \\
$\mathrm{~A}_{\text {mut }} \mathrm{A}_{\mathrm{wt}}$ & $(1+h s)(1-r)$ & $\approx h s-r$ \\
$\mathrm{~A}_{\text {mut }} \mathrm{A}_{\text {mut }}$ & $(1+s)(1-r)$ & $\approx s-r$ \\
$\mathrm{X}_{\mathrm{wt}} \mathrm{X}_{\mathrm{wt}}$ & $(1-r)$ & $-r$ \\
$\mathrm{X}_{\text {mut }} \mathrm{X}_{\mathrm{wt}}$ & $(1+h s)(1-r)$ & $\approx h s-r$ \\
$\mathrm{X}_{\text {mut }} \mathrm{X}_{\text {mut }}$ & $(1+s)(1-r)$ & $\approx s-r$ \\
$\mathrm{X}_{\mathrm{wt}} \mathrm{Y}$ & $(1-r)$ & $-r$ \\
$\mathrm{X}_{\text {mut }} \mathrm{Y}$ & $(1+s)(1-r)$ & $\approx s-r$ \\
\hline
\end{tabular}

Rescue mutations may either be segregating in the standing genetic variation at time zero or appear through new mutation at rate $\mu$ per gamete per generation. We consider these standing variation versus new-mutation cases separately. Populations ultimately have one of two mutually exclusive fates: they become extinct, or they are rescued by mutant alleles.

In haploids, the probability that a single mutation escapes stochastic loss and rescues a population is $P_{\text {rescue }}=$ $1-\exp (-2(s-r)) \approx 2(s-r)$ (Orr and Unckless 2008). The logic underlying this result combines Haldane's (1927) classic $2 s$ approximation for the probability of fixation of a single new beneficial mutation (when $s$ is small) and work by Otto and Whitlock (1997) showing that the probability of fixation of a single new beneficial mutation in a population of changing size is roughly $2(s-r)$. A similar logic suggests that at a diploid autosomal gene, the probability of rescue from a single autosomal mutation is

$$
P_{\text {rescue }}=1-\exp (-2(h s-r)) \approx 2(h s-r),
$$

where we ignore mutant homozygotes (as they are very rare) and assume that the mutation is net beneficial in heterozygotes (see eq. [2] below). The approximation assumes that the quantity $2(h s-r)$ is small. For an autosomal mutation in a heterozygote to be net beneficial, $(1-r)(1+h s)-$ $1 \approx h s-r>0$, or

$$
h>\frac{r}{s}
$$

where the approximation assumes that the product $r h s$ is negligibly small.

Given random mating, small $s$, and an equal sex ratio, the effective selective advantage of an X-linked mutation equals the selective advantage averaged over the two sexes, or

$$
s_{e}=\frac{1}{3}(s-r)+\frac{2}{3}(h s-r)=\frac{1}{3}(s+2 h s-3 r),
$$

where the first term represents the fitness advantage in hemizygous males and the second term represents the fitness advantage in heterozygous females, weighted by the proportion of time the $\mathrm{X}$ finds itself in each sex (Nagylaki 
1979). Note that the equal sex ratio assumption may be dispensable - even with unequal sex ratios, all offspring have a mother and a father, and our effective selection coefficient should remain approximately correct. This leads to the probability of rescue of a single mutation that segregates on the $\mathrm{X}$ chromosome:

$$
P_{\text {rescue } \mid \mathrm{X}}=1-\exp \left(-\frac{2}{3}(s+2 h s-3 r)\right),
$$

again assuming that the mutation is net beneficial (eq. [4]). For X-linked rescue mutations to be net advantageous, $(1 / 3)(1-r)(1+s)+(2 / 3)(1-r)(1+h s)-1 \approx s(1+$ $2 h) / 3-r>0$, or

$$
h>\frac{3 r}{2 s}-\frac{1}{2}
$$

where we again assume that terms involving the products $r s$ and $r h s$ are small enough that they may be ignored safely.

Note that the threshold value of $h$ for autosomal loci is always larger (eq. [2]) than the threshold value of $h$ for X-linked loci (eq. [4]) so long as $r<s$ (which must hold for rescue). This makes good intuitive sense, as autosomal mutations cannot experience any hemizygous benefit in males.

In general, the probability of evolutionary rescue is a function of both $P_{\text {rescue }}$ and the number of rescue mutations that segregate in the standing genetic variation $(m)$, that arise from new mutation, or both. Therefore, the total probability of rescue must be

$$
P_{\text {rescue } \mid m}=1-\left(1-P_{\text {rescue }}\right)^{m} .
$$

In other words, equation (5) provides the probability that, given that $m$ mutations are initially segregating, at least one escapes stochastic loss and rescues the population. Mathematically, this is equivalent to 1 minus the probability that all $m$ mutations are lost. These basic results will allow us to compare analytically autosomal and sex-linked rescue scenarios, albeit approximately.

\section{Simulations}

All analytical results were checked by brute-force forward Monte-Carlo simulations written in R (R Core Team 2018). Each realization was initiated with an appropriate number of homozygous wild-type, heterozygous, and homozygous mutant individuals. Simulations involving new mutation began with only homozygous wild-type individuals, while simulations involving the standing genetic variation began with the deterministic Hardy-Weinberg equilibrium number of individuals for each genotype.

Each generation, matings were chosen randomly and each genotype produced offspring according to a Poisson distribution with their absolute fitness as the parameter. Thus, all genotypes (including the wild type) experienced some stochasticity due to the Poisson distribution of offspring number. Mutations (for the new-mutation case) were initiated on the basis of the mutation rate following a binomial distribution based on the number of wild-type individuals at any given generation.

Each realization continued until either the mutant restored the population size to $10 \%$ of its initial size (which was deemed adequate for a rescue event) or the population became extinct. (When considering adaptation from the standing genetic variation, extinction was said to occur when all rescue mutations were lost: at that point, the population is essentially deterministically doomed.)

\section{Results}

\section{Evolutionary Rescue from New Mutation}

The expected cumulative number of new mutations that arise at an autosomal locus after an environmental change at time $t=0$ but before extinction can be found following the approach of Orr and Unckless (2008), who studied this problem in haploids. The answer is approximately $m_{\mathrm{A}}=2 N_{0} \mu / r$ at a diploid locus. (This assumes that no mutations segregate in the standing genetic variation at time $t=0$.) As there are three-fourths as many X chromosomes as autosomes in a diploid population with equal sex ratios, the analogous expected cumulative number of $\mathrm{X}$ linked mutations is approximately $m_{\mathrm{X}}=(3 / 2) N_{0} \mu / r$.

The total probability of evolutionary rescue from new mutations on the autosomes is therefore:

$$
\begin{aligned}
P_{\text {new } \mid \mathrm{A}} & =1-\left(1-P_{\text {rescue } \mid \mathrm{A}}\right)^{m_{\mathrm{A}}} \\
& \approx 1-\exp \left(-4 N_{0} \mu(h s-r) / r\right) .
\end{aligned}
$$

Note that equation (6) is analogous to equation (10) in Orr and Unckless (2008) and equivalent to equation (23) in Glemin and Ronfort (2013).

For X-linked mutations, the total probability of evolutionary rescue from new mutation is

$$
\begin{aligned}
P_{\text {new } \mid \mathrm{X}} & =1-\left(1-P_{\text {rescue } \mid \mathrm{X}}\right)^{m_{\mathrm{X}}} \\
& \approx 1-\exp \left(-N_{0} \mu(s+2 h s-3 r) / r\right) .
\end{aligned}
$$

Figure $1 A$ shows the probability of rescue from new mutation on both the autosomes and the $\mathrm{X}$ chromosome. From equations (6) and (7) as well as figure $1 A$, it is clear that if rescue involves new mutation, it is more likely to occur on the $\mathrm{X}$ chromosome when

$$
h<\frac{1}{2}+\frac{r}{2 s} .
$$

Equation (8) represents one of our main findings. Note that equation (8) differs from the standard scenario discussed 

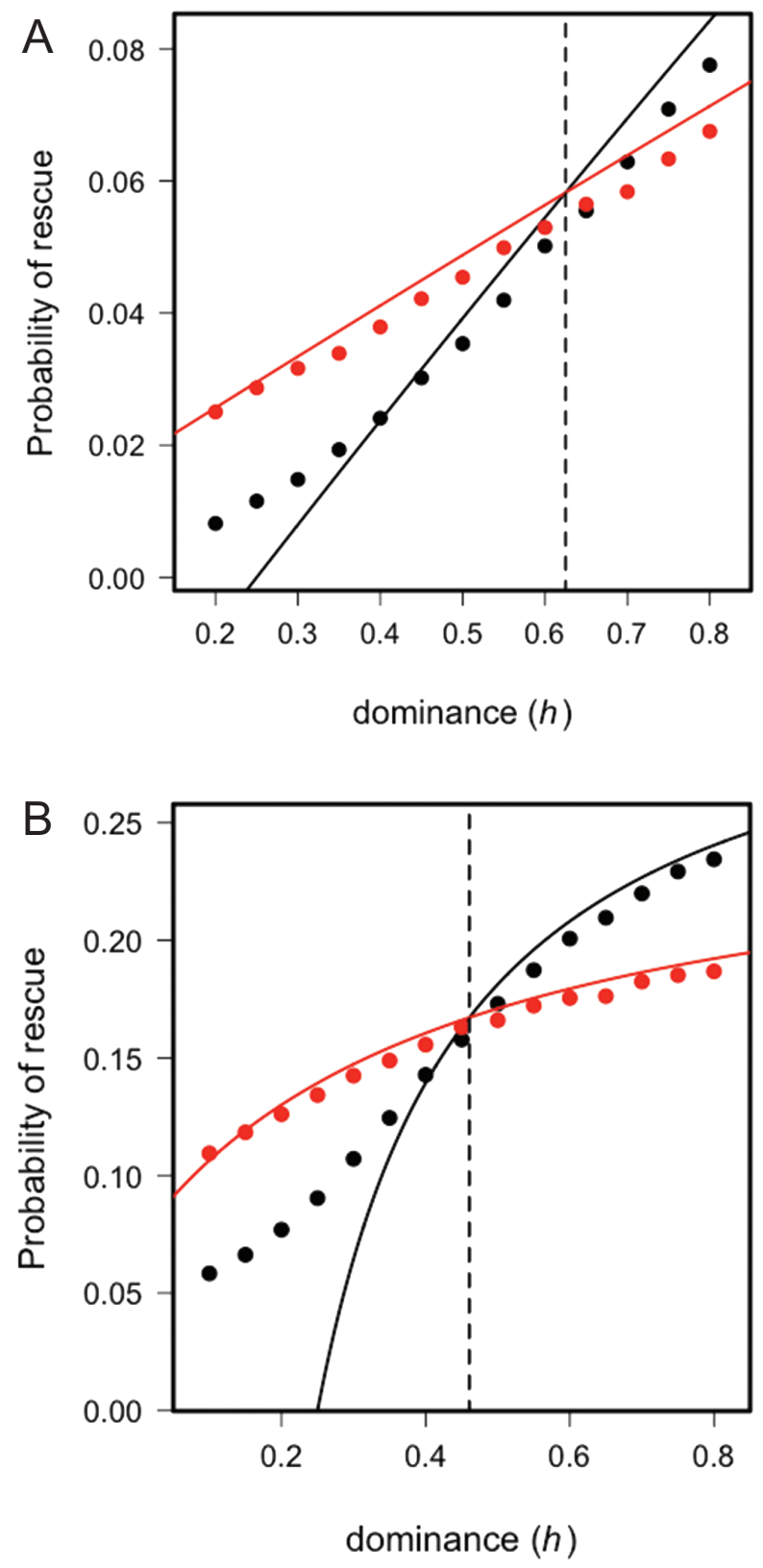

Figure 1: Probability of rescue on autosomes (black) and the X chromosome (red). Lines are analytical results, and points are from simulations. Vertical dashed lines represent the dominance at which rescue from autosomes is more likely than from the $\mathrm{X}$ chromosome. $N_{0}=10,000, s=0.02, r=0.005,100,000$ realizations. $A$, Rescue from new mutation with $\mu=10^{-6}$. $B$, Rescue from standing genetic variation and deterministic mutation-selection balance with $s_{\mathrm{d}}=0.02$ and $\mu=10^{-5}$.

by Charlesworth et al. (1987) in which adaptation from Xlinked vs. autosomal mutations is considered in a population of stable size. In that case, Charlesworth et al. found that adaptation from new mutation is more likely on the $\mathrm{X}$ chro- mosome if $h<1 / 2$. As expected, our critical $h$ in equation (8) collapses to Charlesworth et al.'s result when $r=0$. At the other extreme, equation (8) shows that our critical $h=1$ when $r=s$. Thus, as one might guess, with higher rates of population decline come an increased critical dominance under which the $\mathrm{X}$ chromosome is still more likely to save the population than the autosomes.

The reason that the $\mathrm{X}$ chromosome enjoys a wider parameter space over which it is favored under evolutionary rescue compared with the standard scenario reflects the fact that fitness differences in the rescue scenario must incorporate the parameter $r$. In the standard scenario, the $\mathrm{X}$ chromosome is as likely as the autosomes to contribute to adaptation when $h=1 / 2$. This is because when $h=1 / 2$, the expected fitness advantage of a mutation in males $((1 / 3) s)$ equals the expected fitness advantage of the mutation in heterozygous females. However, for evolutionary rescue to occur, $h$ must be yet larger for the expected fitness advantage in males $((1 / 3)(s-r))$ to equal the expected fitness advantage in heterozygous females $((2 / 3)(h s-r))$. When

$$
h=\frac{1}{2}+\frac{r}{2 s}
$$

(eq. [8]), the fitness advantage in females

$$
\left(\frac{2}{3}\left(\left(\frac{1}{2}+\frac{r}{2 s}\right) s-r\right)=\frac{1}{3}(s-r)\right)
$$

equals the fitness advantage in males.

\section{Evolutionary Rescue from the Standing Genetic Variation}

We now assume that beneficial mutations at the autosomal locus are present in the standing genetic variation at time zero at an initial frequency $p_{\mathrm{A}}$. The analogous frequency at an X-linked locus is $p_{\mathrm{x}}$. Therefore, $m_{\mathrm{A}}=2 N_{0} p_{\mathrm{A}}$ and $m_{\mathrm{X}}=(3 / 2) N_{0} p_{\mathrm{x}}$. Substituting into equation (5) and using an approach similar to that with equation (6), we find

$$
P_{\mathrm{SGV} \mid \mathrm{A}}=1-\left(1-P_{\text {rescue } \mid \mathrm{A}}\right)^{m_{\mathrm{A}}} \approx 1-\exp \left(-4 N_{0} p_{\mathrm{A}}(h s-r)\right) .
$$

For the $\mathrm{X}$ chromosome, we find

$$
\begin{aligned}
P_{\mathrm{SGV} \mid \mathrm{X}} & =1-\left(1-P_{\text {rescue } \mid \mathrm{X}}\right)^{m_{\mathrm{X}}} \\
& \approx 1-\exp \left(-N_{0} p_{\mathrm{X}}(s+2 h s-3 r)\right) .
\end{aligned}
$$

Note that both of these results assume a precise value for the standing genetic variation and do not average across the stationary distribution of frequencies. (This is yet another reason our results are approximate.) Glemin and Ronfort (2013, eq. [21]) consider the stationary distribution (for the autosomes only) and obtain a slightly different result as their second term (in parentheses) incorporates this variation. Note 

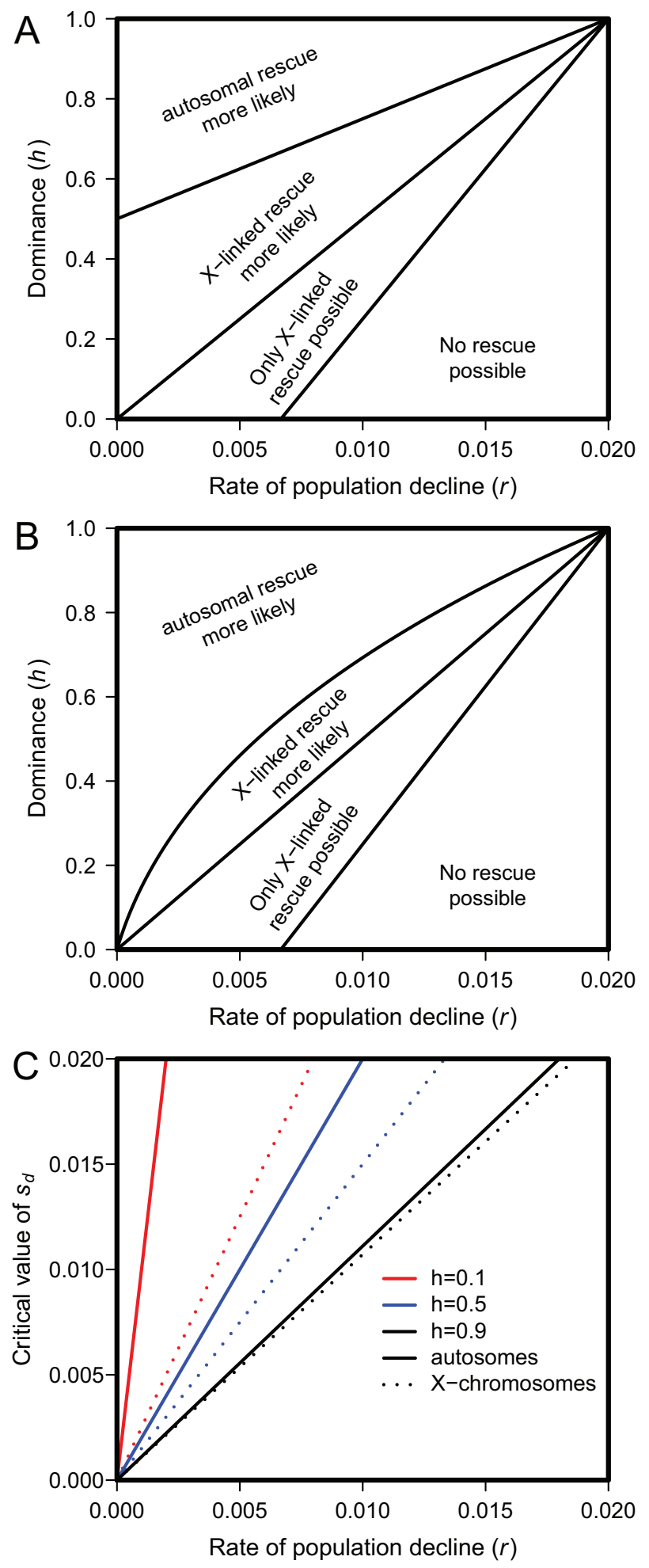

Figure 2: Rescue comparisons (analytical). Shown is the parameter space where rescue is more likely from the autosomes, more likely from the X chromosome, only possible on the X chromosome, or impossible given $s=0.02$. $A, B$, Rescue from new mutation $(A)$ and rescue from standing genetic variation with mutation $(B)$ starting also that both equations (9) and (10) assume that mutants are rare enough that we can ignore mutant homozygotes in the standing genetic variation.

From equations (9) and (10) as well as figure $1 B$, we see that rescue from the standing genetic variation is more likely on the $\mathrm{X}$ chromosome if

$$
h<\frac{4 p_{\mathrm{A}} r-3 p_{\mathrm{X}} r+p_{\mathrm{X}} s}{2 s\left(2 p_{\mathrm{A}}-p_{\mathrm{X}}\right)} .
$$

Equation (11) holds if $2 p_{\mathrm{A}}-p_{\mathrm{x}}>0$, as it does for the deterministic mutation-selection balance case.

It is worth further considering the special case in which the alleles in the standing genetic variation were previously deleterious and segregated at (deterministic) mutationselection equilibrium. In that case, we have $p_{\mathrm{A}}=\mu /\left(h s_{\mathrm{d}}\right)$ and $p_{\mathrm{x}}=3 \mu /\left(s_{\mathrm{d}}(1+2 h)\right)$, where $s_{\mathrm{d}}$ represents the fitness cost of the mutation prior to the environmental change and we assume that dominance $(h)$ is unaffected by the environmental change. In this case, equation (11) becomes

$$
h<\frac{\sqrt{r^{2}+34 r s+s^{2}}-r-s}{4 s} .
$$

Interestingly, the ratio of the cost of the mutation in the previous environment $\left(s_{\mathrm{d}}\right)$ to the mutation rate $(\mu)$ cancels in the course of this calculation, and equation (12) is independent of both. The reason that the ratio $s_{\mathrm{d}} / \mu$ cancels is that it can be extracted from the deterministic $p_{\mathrm{A}}$ and $p_{\mathrm{x}}$ and then canceled when solving for $h$. The biological intuition is that deterministic mutation-selection balance differs on the autosomes versus the $\mathrm{X}$ chromosome because of dominance, not because of the fitness cost of the mutation or the mutation rate. This critical value of $h$ (eq. [12]) ranges from 0 when $r=0$ to 1 when $r=s$ (fig. $2 B$ ). Note, however, that the deterministic mutation-selection balance equation breaks down as $h$ approaches zero (Nei 1968), and we do not consider the strictly recessive case here. Thus, unlike the standard adaptation case, there is parameter space wherein the $\mathrm{X}$ chromosome may be more likely to rescue populations than the autosomes when adapting from standing genetic variation. However, recall that autosomal mutations with $h<r / s$ are not net beneficial anyway. Thus, we can conclude that when mutations on the autosomes are beneficial, they are usually more likely than X-linked mutations to save the population given adaptation from the standing genetic variation.

at deterministic mutation-selection balance. $C$, Cost in previous environment $s_{\mathrm{d}}$ below which rescue from standing genetic variation is more likely than from new mutation on autosomes (solid line) and the $\mathrm{X}$ chromosome (dotted lines) with varying dominance $(s=0.02)$. 
Note that while the probability of rescue is approximately linear in dominance $(h)$ for new mutations (fig. $1 A$ ), the curves showing the probability of rescue bend downward for the standing genetic variation case (fig. $1 B$ ). This is expected for the following reason. As dominance increases, the frequency of the mutation in the standing genetic variation decreases as $h s_{\mathrm{d}}$ grows larger, and therefore the added benefit of increased dominance in the new environment is partially canceled by the increased fitness cost of the mutation in the previous environment. No such effect arises with new mutations.

Orr and Unckless (2014) showed that in haploids, rescue is more likely to involve alleles from the standing genetic variation than from a new mutation if $p>\mu / r$ (where $p$ is the frequency of the mutant in the standing genetic variation). If we assume that the mutation segregates at deterministic haploid mutation-selection equilibrium $\left(p=\mu / s_{\mathrm{d}}\right)$, rescue is more likely from standing genetic variation if $s_{\mathrm{d}}<r$, that is, when the fitness cost in the previous environment is less than the rate of decline in the new environment. For an autosomal diploid locus, the deterministic mutationselection equilibrium is approximately $p=\mu /\left(h s_{\mathrm{d}}\right)$, which means that rescue from the standing genetic variation is more likely when $s_{\mathrm{d}}<r / h$. For an X-linked locus, the analogous inequality is, as in haploids, $p_{\mathrm{X}}>\mu / r$. If we again assume deterministic mutation-selection equilibrium $\left(p_{\mathrm{x}}=\right.$ $\left.3 \mu /\left(s_{\mathrm{d}}(1+2 h)\right)\right)$, rescue from the standing genetic variation is more likely than from new mutation when $s_{\mathrm{d}}<$ $3 r /((1+2 h))$. The critical values of $s_{\mathrm{d}}$ below which rescue from the standing genetic variation is more likely converge as mutations become more fully dominant ( $h \rightarrow 1$; fig. $2 C$ ). But with intermediate dominance, autosomal rescue is more likely to be from the standing genetic variation than $\mathrm{X}$-linked rescue given similar parameters.

\section{Evolutionary Rescue with Intermediate Dominance}

In figure 1, the probability of rescue on autosomes observed in exact computer simulations approaches zero asymptotically, departing from our analytical result. There is a simple explanation for this. On the autosomes, when $h<r / s$, fitness in heterozygotes is less than 1 so that the probability of rescue should be zero, according to the branching-process logic that we have relied on (Haldane 1927). However, if absolute fitness of heterozygotes is close to 1, mutant alleles are likely to remain in the population longer than wild-type alleles (which decline at rate $r$ ). This means that as the population declines, mutant alleles in heterozygotes that persist longer than wild-type alleles (as they are marginally more fit) become more likely to subsequently give rise to mutant homozygotes. This is clear if we consider a simple model (fig. A1, available online) in which the expected number of heterozygous individuals is relatively con- stant but - as homozygous wild-type individuals decline deterministically - the number of homozygous mutants rises. These mutant homozygotes enjoy a full fitness benefit of $(1-r)(1+s)-1 \approx s-r$. Thus, rescue is more likely than predicted by our analytic results, which ignore this subtle effect. Note, however, that this "heterozygote boost" in the probability of rescue generally remains fairly modest, as rare heterozygotes are still usually lost by genetic drift early during population decline. It appears that the same phenomenon occurred in work by Glémin and Ronfort (2013; see their fig. 5), but it was not discussed there as it was not central to that article.

\section{Discussion}

Sex chromosomes often evolve rapidly. This appears partly to reflect the hemizygosity of the $\mathrm{X}$ chromosome in males (i.e., the heterogametic sex). Hemizygosity allows stronger positive selection for beneficial alleles that are incompletely dominant (Vicoso and Charlesworth 2006). Charlesworth et al. (1987) showed that with adaptation from new mutations, adaptive substitution on the $\mathrm{X}$ chromosome should be more frequent than that on an equivalent-sized autosome when beneficial alleles are partially recessive $(h<1 / 2)$. Here, we show that when threatened by extinction, populations are more likely to be rescued evolutionarily by new mutations that are X-linked than autosomal when $h<$ $1 / 2+r / 2 s$. Thus, in the case of evolutionary rescue, the $\mathrm{X}$ chromosome is more likely to contribute to adaptation than in the case of standard nonrescue adaptation. Furthermore, the critical dominance for this X-effect under evolutionary rescue approaches $h=1$ as $r$ approaches $s$. The additional term of $r / 2 s$ reflects the fact that the total fitness associated with both autosomal and X-linked mutations must incorporate the extent of maladaptation in the new environment - and this extent is reflected in $r$.

In the case of adaptation from the standing genetic variation, Orr and Betancourt (2001) found that evolution was slower on the $\mathrm{X}$ chromosome than on an equivalent-sized autosome if alleles were deleterious in the old environment and initially segregated at mutation-selection equilibrium. In the case of evolutionary rescue, the $\mathrm{X}$ chromosome can be more likely to save the population given adaptation from standing genetic variation. However, the relevant comparison of $\mathrm{X}$ chromosomes to autosomes requires both that autosomal mutations are definitely beneficial $(h>r / s)$ and that $h$ is below the threshold in equation (11) or (12). Together, these restrictions can yield a relatively narrow parameter space under which the $\mathrm{X}$ chromosome is favored (fig. 2C). Thus, although a limited range of parameter space may favor evolutionary rescue from the standing genetic variation on the $\mathrm{X}$ chromosome, rescue from the standing genetic variation seems likely to more often involve the 
autosomes. The main reason for this is that given deterministic mutation-selection balance for a previously deleterious allele, the number of copies in the standing genetic variation is always higher on the autosomes than the X chromosome if $h<1$. However, this deficit of mutations on the $\mathrm{X}$ chromosome can be overcome if $h$ is close to $r$.

Our analytic results are approximate. Indeed, our computer simulations reveal that our approximations break down as $r$ approaches $s$. There are at least two reasons for this.

First, as $r$ approaches $s$, autosomal mutations are net beneficial only if $h$ is large. However, this means that heterozygote fitness would be near or below 1, and, as discussed above (and again below), our approximation breaks down when heterozygote fitness is not high enough to rescue the population. Second, as $r$ approaches $s$, the product of the three terms $r h s$ is no longer necessarily negligible relative to other terms (even if $h>r / s$ ), as we assume in our analytic approximations. For example, if $r=0.015, s=$ 0.02 , and $h=0.8$, the approximate fitness excess of a heterozygote is $h s-r=0.001$, while the exact fitness excess is $(1-r)(1+h s)-1=0.00076$, nearly $25 \%$ less. This error arises for each mutation that appears during the course of rescue.

Second, our study also revealed an unforeseen property of evolutionary rescue in diploids. Many mutations with intermediate dominance may improve the fitness of heterozygotes even if the absolute fitness of these individuals remains slightly less than 1 . For such mutations, the branchingprocess approach - which calculates the probability of fixation (and thus of evolutionary rescue) as a function of heterozygous fitness alone-suggests that the probability of rescue is zero. However, computer simulations reveal that such mutations sometimes rescue threatened populations. The reason involves a kind of heterozygote boost effect: a mutation that yields a heterozygous fitness of slightly less than 1 can persist in heterozygous individuals for some number of generations. During this time, the number of wild-type individuals will continue to decline more or less deterministically. But a homozygous-rescue genotype can later arise from the crossing of two heterozygous parents. These rescue homozygotes will now enjoy the full benefit of the rescue mutation - a benefit that yields an absolute fitness greater than 1 - thereby increasing the observed probability of evolutionary rescue above that expected from a branching process approach that considers heterozygotes only.

Our analysis makes several simplifying assumptions that, if violated, might affect our conclusions. First, we assume that, except for ploidy, all is equivalent between the two sexes. However, if mutation rates differ between the sexes our findings could change. Indeed, results from Kirkpatrick and Hall (2004) suggest that male-biased mutation rates could lead to a decreased probability of rescue on the X chromosome compared with the autosomes (but an increased prob- ability of rescue on the $\mathrm{Z}$ chromosome in a $\mathrm{Z} / \mathrm{W}$ system). Similarly, the variance in reproductive success may often be greater in males than in females. This might inflate the ratio of $\mathrm{X}$ chromosome to autosome effective population sizes (Vicoso and Charlesworth 2009), increasing the likelihood of rescue from the $\mathrm{X}$ chromosome. Finally, selection coefficients themselves might differ between the sexes (Connallon et al. 2010). This would likely affect the chances of rescue in the sexes.

Our findings reinforce the notion that evolutionary rescue differs from the standard adaptation scenario in unexpected ways. Rescue from new mutation is relatively more likely to be caused by mutations on the $\mathrm{X}$ chromosome than is true under standard adaptation. The same disparity occurs, although to a lesser extent, for rescue from the standing genetic variation. Finally, we find that rescue mutations with intermediate dominance may be unable to rescue the population on their own but may persist long enough as heterozygotes that they ultimately lead to evolutionary rescue by later forming mutant homozygotes with absolute fitness greater than 1 .

\section{Acknowledgments}

We thank Maria Orive, John Kelly, two anonymous reviewers, and our editor for thoughtful comments about the approach and early versions of the manuscript. This work was supported by grants R00GM114714 and R01AI139154 from the National Institutes of Health to R.L.U. and by funds provided by the University of Rochester to H.A.O.

\section{Literature Cited}

Bell, G. 2008. Selection: the mechanism of evolution. Oxford University Press, New York.

Betrán, E., K. Thornton, and M. Long. 2002. Retroposed new genes out of the X in Drosophila. Genome Research 12:1854-1859.

Charlesworth, B., J. Coyne, and N. Barton. 1987. The relative rates of evolution of sex chromosomes and autosomes. American Naturalist 130:113-146.

Connallon, T., R. M. Cox, and R. Calsbeek. 2010. Fitness consequences of sex-specific selection. Evolution 64:1671-1682.

Glemin, S., and J. Ronfort. 2013. Adaptation and maladaptation in selfing and outcrossing species: new mutations versus standing variation. Evolution 67:225-240.

Haldane, J. B. S. 1927. A mathematical theory of natural and artificial selection, part V: selection and mutation. Mathematical Proceedings of the Cambridge Philosophical Society 23:838-844.

Hedrick, P. W. 2007. Sex: differences in mutation, recombination, selection, gene flow, and genetic drift. Evolution 61:2750-2771.

Hollinger, I., and J. Hermisson. 2017. Bounds to parapatric speciation: a Dobzhansky-Muller incompatibility model involving autosomes, X chromosomes, and mitochondria. Evolution 71:1366-1380.

Kirkpatrick, M., and D. W. Hall. 2004. Male-biased mutation, sex linkage, and the rate of adaptive evolution. Evolution 58:437-440. 
Kirkpatrick, M., and S. Peischl. 2013. Evolutionary rescue by beneficial mutations in environments that change in space and time. Philosophical Transactions of the Royal Society B 368:20120082.

Lasne, C., B. Van Heerwaarden, C. M. Sgrò, and T. Connallon. 2019. Quantifying the relative contributions of the $\mathrm{X}$ chromosome, autosomes, and mitochondrial genome to local adaptation. Evolution 73:262-277.

Mank, J. E., E. Axelsson, and H. Ellegren. 2007. Fast-X on the Z: rapid evolution of sex-linked genes in birds. Genome Research 17:618-624.

Mank, J. E., B. Vicoso, S. Berlin, and B. Charlesworth. 2010. Effective population size and the faster-X effect: empirical results and their interpretation. Evolution 64:663-674.

Nagylaki, T. 1979. Selection in dioecious populations. Annals of Human Genetics 43:143-150.

Nei, M. 1968. The frequency distribution of lethal chromosomes in finite populations. Proceedings of the National Academy of Sciences of the USA $60: 517-524$.

Orr, H. A., and A. J. Betancourt. 2001. Haldane's sieve and adaptation from the standing genetic variation. Genetics 157:875-884.

Orr, H. A., and R. L. Unckless. 2008. Population extinction and the genetics of adaptation. American Naturalist 172:160-169.

- 2014. The population genetics of evolutionary rescue. PLoS Genetics 10:e1004551.

Otto, S. P., and M. C. Whitlock. 1997. The probability of fixation in populations of changing size. Genetics 146:723-733.
Presgraves, D. C. 2008. Sex chromosomes and speciation in Drosophila. Trends in Genetics 24:336-343.

Ramsayer, J., O. Kaltz, and M. E. Hochberg. 2013. Evolutionary rescue in populations of Pseudomonas fluorescens across an antibiotic gradient. Evolutionary Applications 6:608-616.

R Development Core Team. 2018. R: a language and environment for statistical computing. R Foundation for Statistical Computing, Vienna.

Uecker, H. 2017. Evolutionary rescue in randomly mating, selfing, and clonal populations. Evolution 71:845-858.

Uecker, H., and J. Hermisson. 2011. On the fixation process of a beneficial mutation in a variable environment. Genetics 188:915-930.

. 2015. The role of recombination in evolutionary rescue. Genetics 202:721-732.

Uecker, H., S. P. Otto, and J. Hermisson. 2014. Evolutionary rescue in structured populations. American Naturalist 183:E17-E35.

Vicoso, B., and B. Charlesworth. 2006. Evolution on the X chromosome: unusual patterns and processes. Nature Reviews Genetics 7:645-653.

- 2009. Effective population size and the faster-X effect: an extended model. Evolution 63:2413-2426.

Wilson, B., P. Pennings, and D. Petrov. 2017. Soft selective sweeps in evolutionary rescue. Genetics 205:1573-1586.

Associate Editor: Tim Connallon Editor: Russell Bonduriansky

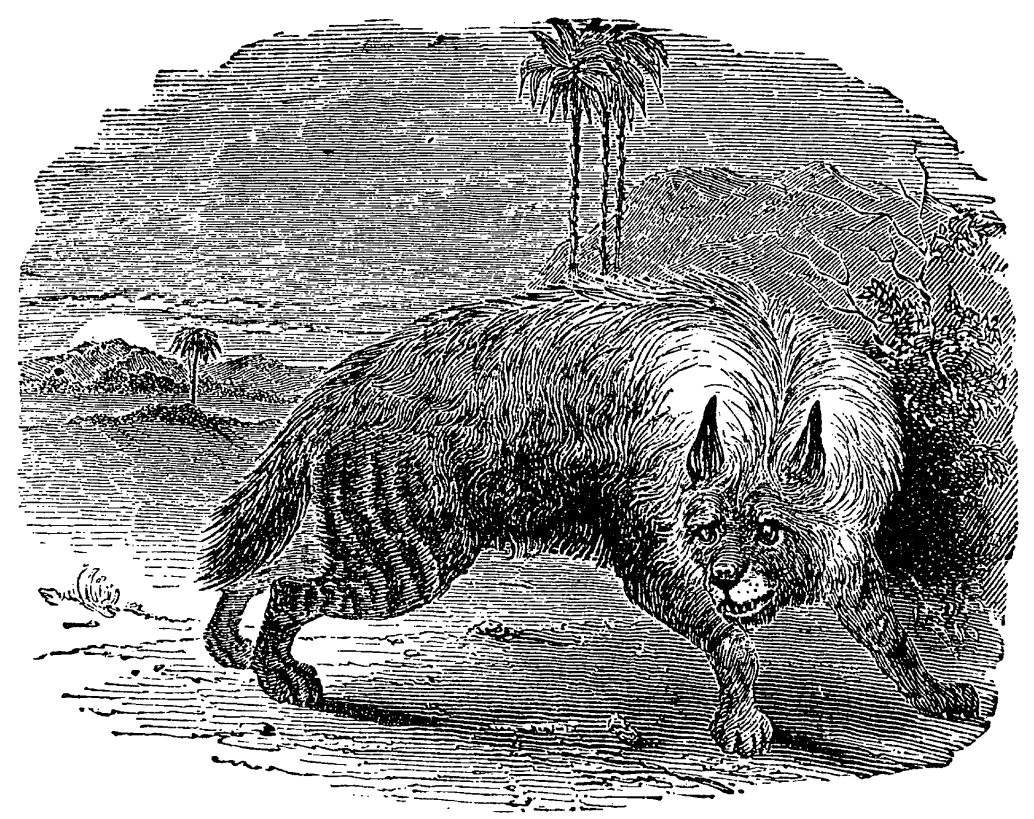

"The hyenas . . . are admirably fitted for their work as scavengers; for they not only devour the soft parts of animals, but their large, blunt premolar teeth and the powerful muscles of their jaws enable them to crush and eat the bones of even very large animals." From "A Few Words about Scavengers" by Sanborn Tenney (The American Naturalist, 1877, 11:129-135). 